\title{
On the core of ideals
}

\author{
Craig Huneke and Ngô Viêt Trung
}

\begin{abstract}
This paper studies the core of an ideal in a Noetherian local or graded ring. By definition, the core of an ideal is the intersection of all reductions of the ideal. We provide computational formulae for the determination of the core of a graded ring, meaning the core of the unique homogeneous maximal ideal. We then apply the formulae to give answers to several questions raised by Corso, Polini and Ulrich. We are also able to answer in the positive a conjecture raised by these three authors concerning a closed formula for the core. We give a positive answer to their question in the case in which the ring is Cohen-Macaulay with a residue field of characteristic 0 , and in the case the ideal is equimultiple.
\end{abstract}

\section{Introduction}

Let $I$ be an ideal in a Noetherian ring. An ideal $J \subseteq I$ is called a reduction of $I$ if there is a positive number $n$ such that $J I^{n}=I^{n+1}$. In other words, $J$ is a reduction of $I$ if and only if $I$ is integrally dependent on $J$ (see [NR54]). The core of $I$, denoted by core $(I)$, is defined to be the intersection of all reductions of $I$.

The core of ideals was first studied by Rees and Sally [RS88], partly due to its connection to the theorem of Briançon and Skoda. Later, Huneke and Swanson [HS95] determined the core of integrally closed ideals in two-dimensional regular local rings and showed a close relationship to Lipman's adjoint ideal. Recently, Corso, Polini and Ulrich [CPU01, CPU02] gave explicit descriptions for the core of certain ideals in Cohen-Macaulay local rings, extending the result of [HS95]. In these two papers, several questions and conjectures were raised which provided motivation for our work. More recently, Hyry and Smith [HS02] have shown that the core and its properties are closely related to a conjecture of Kawamata on the existence of sections for numerically effective line bundles which are adjoint to an ample line bundle over a complex smooth algebraic variety, and they generalize the result in [HS95] to arbitrary dimension and more general rings. Nonetheless, there are many unanswered questions on the nature of the core. One reason is that it is difficult to determine the core and there are relatively few computed examples.

Our focus in this paper is in effective computation of the core with an eye to partially answering some questions raised in [CPU01, CPU02]. A first approach to understanding the core was given by [RS88]. For an ideal $I$ in a local Noetherian $\operatorname{ring}(R, \mathfrak{m})$ having analytic spread $\ell$, one can take $\ell$ generic generators of $I$ in a ring of the form $R\left[U_{i j}\right]_{\mathfrak{m} R\left[U_{i j}\right]}$ which generate an ideal $\mathcal{Q}$. This ideal is then a generic minimal reduction of the extended ideal and a natural question is whether $\operatorname{core}(I)=\mathcal{Q} \cap R$. In [CPU01, Theorem 4.7] this equality is proved under special conditions: namely, if $R$ is a local Cohen-Macaulay ring with infinite residue field, and $I$ satisfies $G_{\ell}$ and is weakly $(\ell-1)$-residually $S_{2}$. While these conditions look somewhat technical, every $\mathfrak{m}$-primary ideal satisfies

Received 22 October 2002, accepted in final form 2 December 2003, published online 1 December 2004.

2000 Mathematics Subject Classification $13 \mathrm{~A} 02$.

Keywords: minimal reduction, core, generic element.

The first author was partially supported by the NSF. The second author is partially supported by the National Basic Research Program of Vietnam.

This journal is (C) Foundation Compositio Mathematica 2005. 


\section{Huneke And N. V. TRung}

the conditions. In particular the core of an m-primary ideal in a local Cohen-Macaulay ring can be computed as the contraction of a generic reduction. In [CPU01, Example 4.11] they give an example to show this is not true if $R$ is not Cohen-Macaulay. Under the same conditions, it is also shown in [CPU01, Theorem 4.8] that, if $R \rightarrow R^{\prime}$ is a flat local homomorphism of CohenMacaulay local rings with infinite residue fields, $I$ is an ideal of $R$ of analytic spread $\ell, I$ and $I R^{\prime}$ are $G_{\ell}$ and universally weakly $(\ell-1)$-residually $S_{2}$, then $\operatorname{core}\left(I R^{\prime}\right)=\operatorname{core}(I) R^{\prime}$. They raise as questions whether or not $\operatorname{core}(I) \subseteq \mathcal{Q} \cap R$ in general and whether or not for arbitrary flat local homomorphisms core $(I) R^{\prime} \subseteq \operatorname{core}\left(I R^{\prime}\right)$. We are able to give partial answers to both of these questions (see the discussion below).

In [CPU02, Conjecture 5.1] the following very general conjecture was made concerning how to calculate the core.

Conjecture 0.1. Let $R$ be a local Cohen-Macaulay ring with infinite residue field. Let $I$ be an $R$-ideal of analytic spread $\ell$ that satisfies $G_{\ell}$ and is weakly $(\ell-1)$-residually $S_{2}$. Let $J$ be a minimal reduction of $I$ and let $r$ denote the reduction number of $I$ with respect to $J$. Then

$$
\operatorname{core}(I)=\left(J^{r}: I^{r}\right) I=\left(J^{r}: I^{r}\right) J=J^{r+1}: I^{r} .
$$

An interesting case of this conjecture occurs when $I$ is an equimultiple ideal. In this case the conditions $G_{\ell}$ and weakly $(\ell-1)$-residually $S_{2}$ are automatically satisfied, so the conjecture applies to all such ideals. In [HS02, Theorem 1.3.3], it is shown that the core of $I$ is equal to $J^{n+1}: I^{n}$ for $n \gg 0$ provided $I$ is equimultiple, $R$ contains the rational numbers, and $R[I t]$ is Cohen-Macaulay. We are able to verify part of Conjecture 0.1 for equimultiple ideals in local rings with characteristic-0 residue field, namely that $\operatorname{core}(I)=J^{r+1}: I^{r}$. We have been informed that this result has been obtained independently by Polini and Ulrich when $\operatorname{dim} R=1$ or when $R$ is a Gorenstein ring [PU04]. The one-dimensional case of this conjecture also follows from the work of Hyry and Smith [HS02].

Another main result of this paper is a closed formula for the graded core of the maximal graded ideal of standard graded algebras over a field. Let $A$ be a standard graded algebra over a field $k$. A reduction of $A$ is a graded ideal $Q$ generated by linear forms such that $Q_{n}=A_{n}$ for all large $n$. Similarly as in the local case, we define the core of $A$, denoted by core $(A)$, to be the intersection of the (minimal) reductions of $A$. In other words, $\operatorname{core}(A)$ is the intersection of the graded reductions of the irrelevant ideal of $A$. The importance of the graded core of a homogeneous ideal has recently been shown by Hyry and Smith [HS02].

If $k$ is an infinite field, every minimal reduction of $A$ can be considered as a specialization of an ideal $Q_{u}$ generated by generic linear forms in a polynomial ring $A[u]$. The parameter space of specializations which are minimal reductions of $A$ has been described explicitly in [Tru03]. This leads us to the interesting problem of determining the intersection of the specializations of a graded module over $k[u]$ upon a given locus. We are able to give an effective solution to this problem when $k$ is an algebraically closed field (Corollary 1.4). Combining this result with a stratification of the parameter space of minimal reductions of $A$ we get a closed formula for $\operatorname{core}(A)$ in terms of the generic ideal $Q_{u}$ (Theorem 1.6). This formula allows us to study basic properties of the graded core. For instance, we can show that, in general, $\operatorname{core}\left(A \otimes_{k} E\right) \neq \operatorname{core}(A) \otimes_{k} E$, where $E$ is a field extension of $k$ (Example 1.8).

Our formula for the graded core of the maximal irrelevant ideal is perhaps more interesting when the base ring is not Cohen-Macaulay. In terms of computation, one can always intersect arbitrary minimal reductions and hope for stabilization. This strategy is particularly effective in the Cohen-Macaulay case where there is a bound for how many such intersections are needed [CPU01]. However, in the non-Cohen-Macaulay case, one has to take into account special minimal reductions which can not be chosen arbitrarily, due to the stratification in our formula. 


\section{ON THE CORE OF IDEALS}

The results on the core of graded algebras will be found in $\S 1$. Section 2 largely deals with the core of ideals in local rings. We construct counter-examples to some open questions on the core raised in [CPU01] and [Tru03]. In particular, we show that the equation $\operatorname{core}\left(I R^{\prime}\right)=\operatorname{core}(I) R^{\prime}$ does not hold for an arbitrary flat local homomorphism $R \rightarrow R^{\prime}$ of Cohen-Macaulay local rings (Example 2.4). In $\S 3$ we settle Conjecture 0.1 in the affirmative for equimultiple ideals in Cohen-Macaulay rings with characteristic-0 residue field (Theorem 3.7). In particular, we can prove in the one-dimensional case that core $(I)=I K$, where $K$ is the conductor of $R$ in the blowing-up ring at $I$ (Theorem 3.2).

\section{Core of graded algebras}

Let $A=\bigoplus_{n \geqslant 0} A_{n}$ be a standard graded algebra over an infinite field $k$ with $d=\operatorname{dim} A$. Let $x_{1}, \ldots, x_{m}$ be linear forms which generate the vector space $A_{1}$. For every point $\alpha=\left(\alpha_{i j} \mid i=\right.$ $1, \ldots, d, j=1, \ldots, m) \in \mathbb{P}_{k}^{N}, N:=d m-1$, we will denote by $Q_{\alpha}$ the ideal generated by $d$ linear forms

$$
y_{i}=\alpha_{i 1} x_{1}+\cdots+\alpha_{i m} x_{m} \quad(i=1, \ldots, d) .
$$

We may consider $Q_{\alpha}$ as a point of the Grassmannian $G(d, m)$. However, for computational purposes we will study the properties of $Q_{\alpha}$ in terms of $\alpha$.

Let $u=\left(u_{i j} \mid i=1, \ldots, d, j=1, \ldots, m\right)$ be a family of $N+1$ indeterminates. Consider $d$ generic elements

$$
z_{i}=u_{i 1} x_{1}+\cdots+u_{i m} x_{m} \quad(i=1, \ldots, d) .
$$

For every integer $n \geqslant 0$ fix a basis $\mathcal{B}_{n}$ for the vector space $A_{n}$. For $n \geqslant 1$ we write every element of the form $z_{i} f, i=1, \ldots, d, f \in \mathcal{B}_{n-1}$, as a linear combination of the elements of $\mathcal{B}_{n}$. Let $M_{n}$ denote the matrix of the coefficients of these linear combinations. Then $M_{n}$ is a matrix with entries in $k[u]$. For every integer $t \geqslant 0$ let $I_{t}\left(M_{n}\right)$ denote the ideal of $k[u]$ generated by the $t$-minors of $M_{n}$. Put $h_{n}=\operatorname{dim}_{k} A_{n}$. Let $V_{n}$ be the zero locus of $I_{h_{n}}\left(M_{n}\right)$ in $\mathbb{P}_{k}^{N}$. It was shown in [Tru03, Theorem 2.1(i)] that $\mathbb{P}_{k}^{N}=V_{0} \supseteq V_{1} \supseteq \cdots \supseteq V_{n} \supseteq \cdots$ is a non-increasing sequence of projective varieties. This sequence must be stationary for $n$ large enough. Hence we can introduce the number

$$
r:=\max \left\{n \mid V_{n} \neq V_{n+1}\right\} .
$$

By [Tru03, Theorem 2.1(ii)] we get the following parametric characterization for the minimal reductions of $A$.

Proposition 1.1. The ideal $Q_{\alpha}$ is a minimal reduction of $A$ if and only if $\alpha \notin V_{r+1}$.

The number $r$ introduced above has a clear meaning. For every minimal reduction $Q$ of $A$ let $r_{Q}(A)$ denote the largest number $n$ such that $Q_{n} \neq A_{n}$. Then $r_{Q}(A)$ is called the reduction number of $Q$. If $Q=\left(x_{1}, \ldots, x_{d}\right)$, then $r_{Q}(A)$ is the maximum degree of the minimal generators of $A$ as a graded module over its Noether normalization $k\left[x_{1}, \ldots, x_{d}\right]$ [Vas96]. The supremum of the reduction numbers of minimal reductions of $A$ is called the big reduction number of $A$ [Vas99] and we will denote it by $\operatorname{br}(A)$. It was shown in [Tru03, Corollary 2.3] that

$$
\operatorname{br}(A)=r .
$$

Upper bounds for $\operatorname{br}(A)$ in terms of other invariants of $A$ can be found in [Tru87], [Vas96] and [Vas99]. We note that it is obvious that

$$
\mathfrak{m}^{b r(A)+1} \subseteq \operatorname{core}(A) .
$$

Let $Q_{u}$ be the ideal of $A[u]$ generated by the generic elements $z_{1}, \ldots, z_{d}$. It is clear that every minimal reduction $Q_{\alpha}$ is obtained from $Q_{u}$ by the substitution $u$ to $\alpha$. If we view $A[u]$ as a graded 


\section{Huneke And N. V. TRung}

algebra over $k[u]$, then every form of a fixed degree $n$ of $Q_{\alpha}$ is the evaluation of a form of degree $n$ in $u$ of $Q_{u}$ at $\alpha$. Since $A[u]_{n}$ is a free module of rank $h_{n}$ over $k[u]$, we may represent every form of degree $n$ in $u$ of $Q_{u}$ as a vector of $h_{n}$ polynomials in $k[u]$. If we evaluate these polynomials at $\alpha$, we will get a vector which represents a form of degree $n$ of $Q_{\alpha}$ and all forms of $Q_{\alpha}$ are obtained in this way. By Proposition 1.1, a form of degree $n$ belongs to core $(A)$ if and only if, for all $\alpha \notin V_{r+1}$, it is the evaluation of some form of degree $n$ in $u$ of $Q_{u}$ at $\alpha$. Therefore, to compute core $(A)$ we need to consider the following problem.

Problem. Let $E$ be a graded submodule of a free module $F=k[u]^{h}, h \geqslant 1$. Consider the elements of $E$ as vectors of $h$ polynomials in $k[u]$. Given a projective variety $V \subset \mathbb{P}_{k}^{N}$, determine the set

$$
\operatorname{core}_{V}(E):=\bigcap_{\alpha \notin V} E_{\alpha},
$$

where $E_{\alpha} \subseteq k^{h}$ denotes the vector space generated by all vectors obtained from vectors of $E$ by the substitution $u$ to $\alpha$.

This problem can be effectively solved if $k$ is an algebraically closed field. In fact our solution can be phrased quite generally and computationally solves how to compute $\bigcap_{\mathfrak{m}} \mathfrak{m} M$ where $M$ is a finitely generated $R$-module, $R$ is a Noetherian Jacobson ring, and the intersection runs over all maximal ideals in an open set of $\operatorname{Spec}(R)$. Recall that $R$ is said to be Jacobson if every prime ideal is the intersection of the maximal ideals containing it. Explicitly, we have the following proposition.

Proposition 1.2. Let $R$ be a Noetherian Jacobson ring. Let $M$ be a finitely generated $R$-module with a presentation

$$
G \stackrel{\phi}{\longrightarrow} F \longrightarrow M \longrightarrow 0
$$

where $G$ and $F$ are finitely generated free $R$-modules. Let $I_{j}$ be the ideal of $j$-minors of $\phi$, where, by convention, $I_{0}=R$. Fix an ideal $J$ in $R$, and let $D(J) \subseteq \operatorname{mspec}(R)$ be the open set in the maximal spectrum of $R$ given by all maximal ideals not containing $J$. Then,

$$
\bigcap_{\mathfrak{m} \in D(J)} \mathfrak{m} M=\bigcap_{t \geqslant 1}\left(\sqrt{I_{t}}\right) M:_{M} J I_{t-1}
$$

Proof. We first reduce to the case $J=R$. Let $v \in \bigcap_{\mathfrak{m} \in D(J)} \mathfrak{m} M$. Let $x \in J$ be arbitrary. Then $x v \in \bigcap_{\mathfrak{m} \in \operatorname{mspec}(R)} \mathfrak{m} M$. We assume we have proved the proposition in the case that $J=R$, in which case $D(J)=\operatorname{mspec}(R)$. Then

$$
x v \in \bigcap_{t \geqslant 1}\left(\sqrt{I_{t}}\right) M:_{M} I_{t-1} .
$$

Since $x$ is arbitrary in $J$, it follows that

$$
v \in \bigcap_{t \geqslant 1}\left(\sqrt{I_{t}}\right) M: M J I_{t-1}
$$

Conversely, suppose that

$$
v \in \bigcap_{t \geqslant 1}\left(\sqrt{I_{t}}\right) M: M J I_{t-1}
$$

For all $x \in J$,

$$
x v \in \bigcap_{t \geqslant 1}\left(\sqrt{I_{t}}\right) M: M I_{t-1},
$$

so assuming the case in which $J=R$, we have that $x v \in \bigcap_{\mathfrak{m} \in \operatorname{mspec}(R)} \mathfrak{m} M$. If $\mathfrak{m} \in D(J)$, then $x v \in \mathfrak{m} M$ will imply that $v \in \mathfrak{m} M$, because $M / \mathfrak{m} M$ is a vector space over $R / \mathfrak{m}$, and the image of $x$ 


\section{ON THE CORE OF IDEALS}

in $R / \mathfrak{m}$ is a unit. Thus, $v \in \bigcap_{\mathfrak{m} \in D(J)} \mathfrak{m} M$. It remains to prove the proposition in the case in which $J=R$, which we henceforth assume.

Let $\mathfrak{m}$ be a maximal ideal of $R$. We adopt the following notation: for a module or element, we write an overline for the image after tensoring with $R / \mathfrak{m}$.

We prove that

$$
\bigcap_{t \geqslant 1}\left(\sqrt{I_{t}}\right) M:_{M} I_{t-1} \subseteq \bigcap_{\mathfrak{m} \in \operatorname{mspec}(R)} \mathfrak{m} M .
$$

Let $v$ be an element in $\bigcap_{t \geqslant 1}\left(\sqrt{I_{t}}\right) M:_{M} I_{t-1}$. Let $\mathfrak{m}$ be an arbitrary maximal ideal of $R$, and set $r$ equal to the rank of $\bar{\phi}$. Then $I_{r+1}(\phi) \subseteq \mathfrak{m}$, and $I_{r}$ is not contained in $\mathfrak{m}$. Choose an element $c \notin \mathfrak{m}$ such that $c \in I_{r}$. By assumption, we have that $c v \in \sqrt{I_{r+1}} M$, and hence $\bar{c} \cdot \bar{v}=0$ in $\bar{M}=M / \mathfrak{m} M$. But $\bar{c}$ is a non-zero element of the field $R / \mathfrak{m}$, and thus $\bar{v}=0$, i.e. $v \in \mathfrak{m} M$.

To finish the proof we show the opposite containment, i.e.

$$
\bigcap_{\mathfrak{m} \in \operatorname{mspec}(R)} \mathfrak{m} M \subseteq \bigcap_{t \geqslant 1}\left(\sqrt{I_{t}}\right) M:_{M} I_{t-1}
$$

Let $v \in \bigcap_{\mathfrak{m} \in \operatorname{mspec}(R)} \mathfrak{m} M$. Choose a lifting $u \in F$ of $v$. By assumption, for all maximal ideals $\mathfrak{m}$, $\bar{v}=0$, so that $\bar{u}$ is in the image of $\bar{\phi}$. We claim that for all $t, I_{t-1} v \subseteq\left(\sqrt{I_{t}}\right) M$. To prove this it suffices to prove that for all $t, I_{t-1} u \subseteq\left(\sqrt{I_{t}}\right) F+\operatorname{Im}(\phi)$. Assume this is not true for some $t$, which we fix. Replace $R$ by $R / \sqrt{I_{t}}$, and $M$ by $M /\left(\sqrt{I_{t}}\right) M$. For all maximal ideals $\mathfrak{m}$ of $R / \sqrt{I_{t}}$, we have that the image of $v$ in $M /\left(\sqrt{I_{t}}\right) M$ is in $\mathfrak{m}\left(M /\left(\sqrt{I_{t}}\right) M\right)$, and to achieve a contradiction, it suffices to prove that $v I_{t-1}=0$. Henceforth we assume that $\sqrt{I_{t}}=0$. In this case the rank of $\phi$ is at most $t-1$. Let $G=R^{s}$ and $F=R^{h}$. Adjoin $u$ to the matrix $\phi$ to get an $h$ by $s+1$ matrix $\psi$ whose last column is $u$. For all maximal ideals $\mathfrak{m}$, the rank of $\bar{\psi}$ is the same as the rank of $\bar{\phi}$. Hence the rank of $\bar{\phi}$ and thus the rank of $\bar{\psi}$ is at most $t-1$. From Lemma 1.3 below it follows that $I_{t}(\psi)=0$. Let $e_{1}, \ldots, e_{s}$ be the given basis of $G$. If we adjoin the row $\left(\phi\left(e_{1}\right), \ldots, \phi\left(e_{s}\right), u\right)$ to $\psi$ to get a new matrix, then all new $t$-minors (which involve the row $\left.\left(\phi\left(e_{1}\right), \ldots, \phi\left(e_{s}\right), u\right)\right)$ vanish. Expanding these minors we see that the product of any $(t-1)$-minor of $\phi$ with $u$ can be expressed as a linear combination of $\phi\left(e_{1}\right), \ldots, \phi\left(e_{s}\right)$. Therefore, $I_{t-1}(\phi) u \in \operatorname{Im}(\phi)$, proving this direction.

Lemma 1.3. Let $R$ be a reduced Jacobson ring, and let $\phi: R^{s} \longrightarrow R^{h}$ be a homomorphism of free $R$-modules. Then $\operatorname{rank}(\phi)=\max \{\operatorname{rank}(\bar{\phi})\}$ where the maximum is taken over all maximal ideals $\mathfrak{m}$ of $R$, and where $\bar{\phi}$ denotes the map from $(R / \mathfrak{m})^{s} \longrightarrow(R / \mathfrak{m})^{h}$ induced by $\phi$.

Proof. Clearly the maximum is at most the rank of $\phi$. Set $r=\operatorname{rank}(\phi)$. Then the $(r+1)$-size minors of $\phi$ are zero, and there is a non-zero $r \times r$ minor. Since $R$ is reduced and Jacobson, there is a maximal ideal $\mathfrak{m}$ which does not contain $I_{r}(\phi)$. Passing to $R / \mathfrak{m}$ gives that $r=\operatorname{rank}(\bar{\phi})$.

Now we use Proposition 1.2 to study the problem above. Let $E$ be a graded submodule of a free module $F=k[u]^{h}$ for $h \geqslant 1$. Choose a set of generators $g_{1}, \ldots, g_{s}$ for $E$. Let $B$ denote the $h \times s$ matrix of the coordinates of $g_{1}, \ldots, g_{s}$. For every integer $t \geqslant 0$ let $I_{t}$ be the ideal of $k[u]$ generated by the $t$-minors of $B$. We let $M=F / E$, and denote the canonical projection of $F$ onto $M$ by $\pi$.

Corollary 1.4. Assume that $k$ is an algebraically closed field. Let $J$ be an ideal of $k[u]$ such that $\sqrt{J}$ is the defining ideal of $V$. Then

$$
\operatorname{core}_{V}(E)=\bigcap_{t \geqslant 1}\left[\left(E+\sqrt{I_{t}} F\right): J I_{t-1}\right] \cap k^{r} .
$$

Proof. Let $f$ be an arbitrary vector in $\operatorname{core}_{V}(E)$. We think of $f$ as living in $k[u]^{h}$ as a vector of constants. Note that $\alpha \notin V$ if and only if the maximal ideal $\mathfrak{m}_{\alpha} \in D(J)$. Then $f \in E_{\alpha}$ if and only if 


\section{Huneke And N. V. TRung}

$\pi(f) \in \mathfrak{m}_{\alpha} M$. Hence by Proposition 1.2 , we have that $f \in \operatorname{core}_{V}(E)$ if and only if $f \in \bigcap_{\alpha \notin V} E_{\alpha}$, if and only if

$$
\pi(f) \in \bigcap_{\mathfrak{m}_{\alpha} \in D(J)} \mathfrak{m}_{\alpha} M=\bigcap_{t \geqslant 1}\left(\sqrt{I_{t}}\right) M:_{M} J I_{t-1},
$$

if and only if

$$
f \in \bigcap_{t \geqslant 1}\left[\left(E+\sqrt{I_{t}} F\right): J I_{t-1}\right] .
$$

The above corollary allows us to compute the graded pieces of $\operatorname{core}(A)$. But what we need is a closed formula for the whole ideal core $(A)$. Such a formula can be found by taking into account all possible Hilbert functions of minimal reductions of $A$. First, we shall describe the parameter space of minimal reductions with a given Hilbert function.

Let $n$ and $t$ be fixed positive integers. We denote by $V_{n, t}$ the zero locus of $I_{t}\left(M_{n}\right)$ in $\mathbb{P}_{k}^{N}$, where $I_{t}\left(M_{n}\right)$ is the ideal of $k[u]$ generated by the $t$-minors of the matrix $M_{n}$ introduced before Proposition 1.1. Note that $V_{n, 1} \subseteq V_{n, 2} \subseteq \cdots V_{n, t} \subseteq \cdots$ is a non-decreasing sequence of subschemes of $\mathbb{P}_{k}^{N}$ and that $V_{n}=V_{n, h_{n}}$, where $h_{n}=\operatorname{dim}_{k} A_{n}$.

LEMMA 1.5. With the above notation we have

$$
V_{n, t+1} \backslash\left(V_{n, t} \cup V_{r+1}\right)=\left\{\alpha \in \mathbb{P}_{k}^{N} \mid Q_{\alpha} \text { is a minimal reduction of } A \text { with } \operatorname{dim}_{k}\left(Q_{\alpha}\right)_{n}=t\right\} \text {. }
$$

Proof. Let $M_{n}(\alpha)$ denote the matrix obtained from $M_{n}$ by the substitution $u$ to $\alpha$. By the definition of $M_{n}$ we have $\operatorname{dim}_{k}\left(Q_{\alpha}\right)_{n}=\operatorname{rank} M_{n}(\alpha)$. Hence $\operatorname{dim}_{k}\left(Q_{\alpha}\right)_{n}=t$ since $\alpha \in V_{n, t+1} \backslash V_{n, t}$. By Proposition 1.1, $Q_{\alpha}$ is a minimal reduction of $A$ if and only if $\alpha \notin V_{r+1}$. The conclusion is immediate.

Let $Q_{\alpha}$ be an arbitrary minimal reduction of $A$. For $n \geqslant r+1$ we have $\left(Q_{\alpha}\right)_{n}=A_{n}$ by [Tru03, Theorem 2.1(ii)], hence $\operatorname{dim}_{k}\left(Q_{\alpha}\right)_{n}$ is independent of the choice of $Q_{\alpha}$. Therefore, the Hilbert function of $Q_{\alpha}$ is determined by the finite sequence of values $\operatorname{dim}_{k}\left(Q_{\alpha}\right)_{n}, n=1, \ldots, r$.

To every sequence $H=\left\{a_{1}, \ldots, a_{r}\right\}$ of $r$ positive integers we associate a set

$$
V_{H}:=\bigcap_{n=1}^{r}\left(V_{n, a_{n}+1} \backslash\left(V_{n, a_{n}} \cup V_{r+1}\right)\right) .
$$

We call $H$ an admissible sequence if $V_{H} \neq \emptyset$. Let $\mathcal{S}$ be the set of all admissible sequences. By Lemma 1.5 we have

$$
V_{H}=\left\{\alpha \in \mathbb{P}_{k}^{N} \mid Q_{\alpha} \text { is a minimal reduction of } A \text { with } \operatorname{dim}_{k}\left(Q_{\alpha}\right)_{n}=a_{n}, n=1, \ldots, r\right\} .
$$

Therefore we may view $\mathcal{S}$ as the set of all possible Hilbert functions of minimal reductions of core $(A)$.

The next theorem uses the finiteness of $\mathcal{S}$ to give a closed formula for $\operatorname{core}(A)$. We believe this is the first general such formula without conditions on $A$.

Theorem 1.6. Assume that $k$ is an algebraically closed field. Put $J=I_{h_{r+1}}\left(M_{r+1}\right)$. Then

$$
\operatorname{core}(A)=\bigcap_{\left(a_{1}, \ldots, a_{r}\right) \in \mathcal{S}}\left[\left(Q_{u}+\sum_{n=1}^{r} \sqrt{I_{a_{n}+1}\left(M_{n}\right)}\right): J \prod_{n=1}^{r} I_{a_{n}}\left(M_{n}\right)\right] \cap A .
$$

Proof. For brevity let

$$
C:=\bigcap_{\left(a_{1}, \ldots, a_{r}\right) \in \mathcal{S}}\left[\left(Q_{u}+\sum_{n=1}^{r} \sqrt{I_{a_{n}+1}\left(M_{n}\right)}\right): J \prod_{n=1}^{r} I_{a_{n}}\left(M_{n}\right)\right] \cap A .
$$




\section{ON THE CORE OF IDEALS}

We will first show that $A_{n} \subset C$ for $n \geqslant r+1$, which obviously implies that $\operatorname{core}(A)_{n} \subset C$. Write every element of the form $z_{i} f, i=1, \ldots, d, f \in B_{r}$, as a linear combination of the elements of $B_{r+1}$. By definition, $M_{r+1}$ is the matrix of the coefficients of these linear combinations. Since $h_{r+1}=\operatorname{dim}_{k} A_{r+1}=\sharp B_{r+1}$, the product of every $h_{r+1}$-minor of $M_{r+1}$ with an element of $B_{r+1}$ can be written as a linear combination of the elements $z_{i} f$. From this it follows that $J A_{r+1} \subset Q_{u}$. Hence $A_{r+1} \subset\left(Q_{u}: J\right) \cap A \subseteq C$. Since $A$ is generated by the elements of $A_{1}$, this implies $A_{n} \subset\left(A_{r+1}\right) \subseteq C$ for all $n \geqslant r+1$.

Now we will show that $\operatorname{core}(A)_{n} \subset C$ for $n \leqslant r$. By Proposition 1.1 we have

$$
\operatorname{core}(A)_{n}=\bigcap_{\alpha \notin V_{r+1}}\left(Q_{\alpha}\right)_{n} .
$$

Applying Corollary 1.4 we obtain

$$
\bigcap_{\alpha \notin V_{r+1}}\left(Q_{\alpha}\right)_{n} \subset \bigcap_{t \geqslant 0}\left[\left(Q_{u}+\sqrt{I_{t+1}\left(M_{n}\right)}\right): J I_{t}\left(M_{n}\right)\right] \cap A .
$$

For any sequence $\left(a_{1}, \ldots, a_{r}\right) \in \mathcal{S}$ we have

$$
\left(Q_{u}+\sqrt{I_{a_{n}+1}\left(M_{n}\right)}\right): J I_{a_{n}}\left(M_{n}\right) \subseteq\left(Q_{u}+\sum_{n=1}^{r} \sqrt{I_{a_{n}+1}\left(M_{n}\right)}\right): J \prod_{n=1}^{r} I_{a_{n}}\left(M_{n}\right) .
$$

Therefore

$$
\bigcap_{t \geqslant 0}\left[\left(Q_{u}+\sqrt{I_{t+1}\left(M_{n}\right)}\right): J I_{t}\left(M_{n}\right)\right] \cap A \subseteq C .
$$

So we get $\operatorname{core}(A)_{n} \subset C$ for $n \leqslant r$. Summing up we can conclude that $\operatorname{core}(A) \subseteq C$.

It remains to show that $\operatorname{core}(A) \supseteq C$. Let $f$ be an arbitrary element in $C$. By Proposition 1.1 we have to show that $f \in Q_{\alpha}$ for all $\alpha \notin V_{r+1}$. For every positive integer $n \leqslant r$ choose $a_{n}$ to be the unique positive integer with the property $\alpha \in V_{n, a_{n}+1} \backslash V_{n, a_{n}}$. Put $H=\left\{a_{1}, \ldots, a_{r}\right\}$. Then $\alpha \in V_{H}$. Hence $H$ is an admissible sequence. From this it follows that

$$
f J \prod_{n=1}^{r} I_{a_{n}}\left(M_{n}\right) \subseteq Q_{u}+\sum_{n=1}^{r} \sqrt{I_{a_{n}+1}\left(M_{n}\right)} .
$$

Note that $\alpha$ is a zero of $\sum_{n=1}^{r} \sqrt{I_{a_{n}+1}\left(M_{n}\right)}$ and that there exists a polynomial $c(u) \in J \prod_{n=1}^{r}$ $I_{a_{n}}\left(M_{n}\right)$ such that $c(\alpha) \neq 0$. Since $c(u) f \in Q_{u}+\sum_{n=1}^{r} \sqrt{I_{a_{n}+1}\left(M_{n}\right)}$, substituting $u$ to $\alpha$ we get $f \in Q_{\alpha}$. So we have proved that $C \subseteq \operatorname{core}(A)$. The proof of Theorem 1.6 is now complete.

The following example shows that the condition $k$ being an algebraically closed field is necessary in Theorem 1.6.

Example 1.7. Let $A=\mathbb{R}\left[x_{1}, x_{2}\right]=\mathbb{R}\left[X_{1}, X_{2}\right] /\left(X_{1}^{3} X_{2}+X_{1} X_{2}^{3}, X_{2}^{5}\right)$. Then $\operatorname{dim} A=1$. Put $Q_{u}=$ $\left(u_{1} x_{1}+u_{2} x_{2}\right) \subset \mathbb{R}\left[u_{1}, u_{2}, x_{1}, x_{2}\right]$, where $u_{1}, u_{2}$ are two indeterminates. For every $n$ let $\mathcal{B}_{n}$ be the basis of $A_{n}$ which consists of monomials (in $x_{1}, x_{2}$ ) that have the possibly highest rank in the lexicographical order. The matrices $M_{n}$ of the coefficients of the elements of the form $\left(u_{1} x_{1}+u_{2} x_{2}\right) f$, $f \in \mathcal{B}_{n-1}$, written as linear combinations of elements of $\mathcal{B}_{n}$, look as follows:

$$
\begin{aligned}
& M_{1}=\left(\begin{array}{ll}
u_{1} & u_{2}
\end{array}\right), \\
& M_{2}=\left(\begin{array}{ccc}
u_{1} & u_{2} & 0 \\
0 & u_{1} & u_{2}
\end{array}\right),
\end{aligned}
$$


C. Huneke And N. V. TRung

$$
\begin{aligned}
M_{3} & =\left(\begin{array}{cccc}
u_{1} & u_{2} & 0 & 0 \\
0 & u_{1} & u_{2} & 0 \\
0 & 0 & u_{1} & u_{2}
\end{array}\right), \\
M_{4} & =\left(\begin{array}{cccc}
u_{1} & u_{2} & 0 & 0 \\
0 & u_{1} & u_{2} & 0 \\
0 & -u_{2} & u_{1} & 0 \\
0 & -u_{1} & 0 & u_{2}
\end{array}\right), \\
M_{5} & =\left(\begin{array}{ccc}
u_{1} & u_{2} & 0 \\
0 & u_{1} & u_{2} \\
0 & -u_{2} & u_{1} \\
0 & 0 & -u_{1}
\end{array}\right), \\
M_{6} & =\left(\begin{array}{cc}
u_{1} & 0 \\
0 & u_{2} \\
0 & u_{1}
\end{array}\right), \\
M_{7} & =\left(\begin{array}{c}
u_{1} \\
0
\end{array}\right), \\
M_{n} & =\left(u_{1}\right)(n \geqslant 8) .
\end{aligned}
$$

Therefore, $V_{1}=V_{2}=V_{3}=\mathbb{P}_{\mathbb{R}}^{1}, V_{4}=\left\{u_{1}=0\right\} \cup\left\{u_{2}=0\right\}$ and $V_{n}=\left\{u_{1}=0\right\}$ for $n \geqslant 5$. Hence $r=\operatorname{br}(A)=\max \left\{n \mid V_{n} \neq V_{n+1}\right\}=4$ and $J=u_{1}\left(u_{1}, u_{2}\right)^{2}$. Since the big reduction number is 4, it follows that $\left(x_{1}, x_{2}\right)^{5} \subseteq \operatorname{core}(A)$. Since all minimal reductions of $A$ have the form $\left(\alpha_{1} x_{1}+\alpha_{2} x_{2}\right)$ with $\alpha_{1} \neq 0$ and since $A$ is defined by forms of degree $\geqslant 4$, we can easily check that $\operatorname{core}(A)$ has no elements of degree $<4$ and $x_{1}^{4}, x_{1}^{3} x_{2}, x_{1}^{2} x_{2}^{2}, x_{1} x_{2}^{3} \in \operatorname{core}(A)_{4}, x_{2}^{4} \notin \operatorname{core}(A)_{4}$. Therefore, $\operatorname{core}(A)=\left(x_{1}^{4}, x_{1}^{3} x_{2}, x_{1}^{2} x_{2}^{2}\right)$. Note that

$$
\begin{aligned}
& I_{t}\left(M_{n}\right)=\left(u_{1}, u_{2}\right)^{t}, \quad \text { if } t=1, \ldots, n(n \leqslant 3), \\
& I_{t}\left(M_{4}\right)= \begin{cases}\left(u_{1}, u_{2}\right)^{t}, & \text { if } t=1,2,3, \\
u_{1} u_{2}\left(u_{1}^{2}+u_{2}^{2}\right), & \text { if } t=4 .\end{cases}
\end{aligned}
$$

Then there are two admissible sequences $\{1,2,3,3\}$ and $\{1,2,3,4\}$. If Theorem 1.6 holds for the base field $\mathbb{R}$, we would get

$$
\begin{aligned}
\operatorname{core}(A) & =\left[\left(Q_{u}, u_{1} u_{2}\left(u_{1}^{2}+u_{2}^{2}\right)\right): u_{1}\left(u_{1}, u_{2}\right)^{11}\right] \cap\left[Q_{u}: u_{1}^{2} u_{2}\left(u_{1}^{2}+u_{2}^{2}\right)\left(u_{1}, u_{2}\right)^{8}\right] \cap A \\
& =\left(x_{1}^{4}+x_{1}^{2} x_{2}^{2}, x_{1}^{2} x_{2}^{3}, x_{1} x_{2}^{4}\right) \neq\left(x_{1}^{4}, x_{1}^{3} x_{2}, x_{1}^{2} x_{2}^{2}\right),
\end{aligned}
$$

which is a contradiction.

The next example shows that the formula $\operatorname{core}\left(A \otimes_{k} E\right)=\operatorname{core}(A) \otimes_{k} E$ does not hold for arbitrary field extension $E$ of $k$.

Example 1.8. Let $A=\mathbb{R}\left[x_{1}, x_{2}\right]=\mathbb{R}\left[X_{1}, X_{2}\right] /\left(X_{1}^{2} X_{2}+X_{2}^{3}, X_{2}^{4}\right)$. Then $\operatorname{dim} A=1$. Put $Q_{u}=$ $\left(u_{1} x_{1}+u_{2} x_{2}\right) \subset \mathbb{R}\left[u_{1}, u_{2}, x_{1}, x_{2}\right]$, where $u_{1}, u_{2}$ are two indeterminates. For every $n$ let $\mathcal{B}_{n}$ be the basis of $A_{n}$ which consists of monomials (in $x_{1}, x_{2}$ ) that have the possibly highest rank in the lexicographical order. The matrices $M_{n}$ of the coefficients of the elements of the form $\left(u_{1} x_{1}+u_{2} x_{2}\right) f$, $f \in \mathcal{B}_{n-1}$, written as linear combinations of elements of $\mathcal{B}_{n}$, look as follows:

$$
\begin{aligned}
& M_{1}=\left(\begin{array}{ll}
u_{1} & u_{2}
\end{array}\right), \\
& M_{2}=\left(\begin{array}{ccc}
u_{1} & u_{2} & 0 \\
0 & u_{1} & u_{2}
\end{array}\right),
\end{aligned}
$$


ON THE CORE OF IDEALS

$$
\begin{aligned}
& M_{3}=\left(\begin{array}{ccc}
u_{1} & u_{2} & 0 \\
0 & u_{1} & u_{2} \\
0 & -u_{2} & u_{1}
\end{array}\right), \\
& M_{4}=\left(\begin{array}{cc}
u_{1} & u_{2} \\
0 & u_{1} \\
0 & -u_{2}
\end{array}\right), \\
& M_{5}=\left(\begin{array}{cc}
u_{1} & u_{2} \\
0 & u_{1}
\end{array}\right), \\
& M_{6}=\left(\begin{array}{c}
u_{1} \\
0
\end{array}\right), \\
& M_{n}=\left(u_{1}\right) \quad(n \geqslant 7) .
\end{aligned}
$$

So we get $V_{1}=V_{2}=\mathbb{P}_{\mathbb{R}}^{1}$, and $V_{n}=\left\{u_{1}=0\right\}$ for $n \geqslant 3$. Hence $r=\operatorname{br}(A)=\max \left\{n \mid V_{n} \neq V_{n+1}\right\}=2$ and $J=\left(u_{1}^{3}+u_{1} u_{2}^{2}\right)$. As a consequence, $\left(x_{1}, x_{2}\right)^{3} \subseteq \operatorname{core}(A)$, since in general $\mathfrak{m}^{b r}(A)+1 \subseteq \operatorname{core}(A)$. As all minimal reductions of $A$ have the form $\left(\alpha_{1} x_{1}+\alpha_{2} x_{2}\right)$ with $\alpha_{1} \neq 0$ and since $A$ is defined by forms of degree $>2$, one can easily check that $\operatorname{core}(A)$ does not contain any form of degree 2 . Therefore, $\operatorname{core}(A)=\left(x_{1}, x_{2}\right)^{3}$.

The core of $A$ will be changed if we replace $\mathbb{R}$ by $\mathbb{C}$. In this case, we have $V_{1}=V_{2}=\mathbb{P}_{\mathbb{C}}^{1}, V_{3}=$ $\left\{u_{1}=0\right\} \cup\left\{u_{1}= \pm i u_{2}\right\}$, and $V_{n}=\left\{u_{1}=0\right\}$ for $n \geqslant 4$. Hence $r=\operatorname{br}(A)=\max \left\{n \mid V_{n} \neq V_{n+1}\right\}=3$ and $J=u_{1}\left(u_{1}, u_{2}\right)$. Note that

$$
\begin{aligned}
& I_{t}\left(M_{3}\right)=\left(u_{1}, u_{2}\right)^{2}, \quad \text { if } t=1,2, \\
& I_{3}\left(M_{3}\right)=u_{1}\left(u_{1}^{2}+u_{2}^{2}\right) .
\end{aligned}
$$

We can easily verify that there are only two admissible sequences $(1,2,2)$ and $(1,2,3)$. By Theorem 1.6 we get

$$
\begin{aligned}
\operatorname{core}(A) & =\left[\left(Q_{u}, u_{1}\left(u_{1}^{2}+u_{2}^{2}\right)\right): u_{1}\left(u_{1}, u_{2}\right)^{6}\right] \cap\left[Q_{u}: u_{1}^{2}\left(u_{1}^{2}+u_{2}^{2}\right)\left(u_{1}, u_{2}\right)^{4}\right] \cap A \\
& =\left(x_{1}^{3}+x_{1} x_{2}^{2}, x_{1} x_{2}^{3}\right) \neq\left(x_{1}, x_{2}\right)^{3} .
\end{aligned}
$$

The formula of Theorem 1.6 involves many operations with determinantal ideals. Hence we have tried to find a simpler formula. By the same argument as in the last part of the proof of Theorem 1.6 we always have

$$
\left(Q_{u}: J^{\infty}\right) \cap A \subseteq \operatorname{core}(A),
$$

where $Q_{u}: J^{\infty}$ denotes the set of all elements $f \in A[u]$ such that $f J^{n} \in Q_{u}$ for some positive integer $n$. Since $\sqrt{J}$ is the defining ideal of $V_{r+1}$ and $\mathbb{P}_{k}^{N} \backslash V_{r+1}$ is the parameter space of minimal reductions of $A$, one may raise the question whether core $(A)=\left(Q_{u}: J^{\infty}\right) \cap A$ holds in general. This question has a positive answer in the following case.

Corollary 1.9. Assume that $k$ is an algebraically closed field. If the Hilbert function of every minimal reduction $Q_{\alpha}$ of $A$ does not depend on the choice of $\alpha$, then

$$
\operatorname{core}(A)=\left(Q_{u}: J^{\infty}\right) \cap A .
$$

Proof. It is sufficient to show that $\operatorname{core}(A) \subseteq\left(Q_{u}: J^{\infty}\right) \cap A$. Let $a_{n}=\operatorname{rank} M_{n}, n=1, \ldots, r$, and $H=\left\{a_{1}, \ldots, a_{r}\right\}$. Then $I_{a_{n}}\left(M_{n}\right) \neq 0$ and $I_{a_{n}+1}\left(M_{n}\right)=0$. Hence $V_{n, a_{n}} \neq \mathbb{P}_{k}^{N}$ and $V_{n, a_{n}+1}=\mathbb{P}_{k}^{N}$. It follows that

$$
V_{H}=\mathbb{P}_{k}^{N} \backslash\left(\bigcup_{n=1}^{r} V_{n, a_{n}} \cup V_{r+1}\right) \neq \emptyset .
$$

So $H$ is an admissible sequence. By the independence of Hilbert functions of minimal reductions, the parameter space of minimal reductions equals the parameter space of minimal reductions with 


\section{Huneke and N. V. Trung}

Hilbert functions determined by $H$. Hence we must have $V_{H}=\mathbb{P}_{k}^{N} \backslash V_{r+1}$. This implies $V_{n, a_{n}} \subseteq V_{r+1}$, hence $\sqrt{I_{a_{n}}\left(M_{n}\right)} \supseteq J$ for all $n=1, \ldots, r$. Therefore, applying Theorem 1.6 we get

$$
\operatorname{core}(A)=\left(Q_{u}: J \prod_{n=1}^{r} I_{a_{n}}\left(M_{n}\right)\right) \cap A \subseteq\left(Q_{u}: J^{\infty}\right) \cap A .
$$

The condition on the independence of Hilbert functions of minimal reductions is satisfied if $A$ is a Cohen-Macaulay ring. In this case, one can even show that

$$
\operatorname{core}(A)=\left(Q_{u}: J^{\infty}\right) \cap A=Q_{u} A(u) \cap A,
$$

where $A(u):=A \otimes_{k} k(u)$ (the proof is similar to that of [CPU01, Theorem 4.7(b)] or [Tru03, Corollary 4.6]).

Now we will use Theorem 1.6 to construct a counter-example to the question whether $\operatorname{core}(A)=$ $\left(Q_{u}: J^{\infty}\right) \cap A$ holds in general.

Example 1.10. Let $A=k\left[x_{1}, x_{2}\right]=k\left[X_{1}, X_{2}\right] /\left(X_{1}^{2} X_{2}^{2}, X_{2}^{5}\right)$. Then $\operatorname{dim} A=1$. Put $Q_{u}=\left(u_{1} x_{1}+\right.$ $\left.u_{2} x_{2}\right) \subset k\left[u_{1}, u_{2}, x_{1}, x_{2}\right]$, where $u_{1}, u_{2}$ are two indeterminates. For every $n$ let $\mathcal{B}_{n}$ be the basis of $A_{n}$ which consists of monomials of degree $n$ in $x_{1}, x_{2}$. If we arrange these monomials in lexicographical order, then the matrices $M_{n}$ of the coefficients of the elements of the form $\left(u_{1} x_{1}+u_{2} x_{2}\right) f, f \in \mathcal{B}_{n-1}$, written as linear combinations of elements of $\mathcal{B}_{n}$, look as follows:

$$
\begin{aligned}
& M_{1}=\left(\begin{array}{ll}
u_{1} & u_{2}
\end{array}\right), \\
& M_{2}=\left(\begin{array}{ccc}
u_{1} & u_{2} & 0 \\
0 & u_{1} & u_{2}
\end{array}\right) \text {, } \\
& M_{3}=\left(\begin{array}{cccc}
u_{1} & u_{2} & 0 & 0 \\
0 & u_{1} & u_{2} & 0 \\
0 & 0 & u_{1} & u_{2}
\end{array}\right) \text {, } \\
& M_{4}=\left(\begin{array}{cccc}
u_{1} & u_{2} & 0 & 0 \\
0 & u_{1} & 0 & 0 \\
0 & 0 & u_{2} & 0 \\
0 & 0 & u_{1} & u_{2}
\end{array}\right) \text {, } \\
& M_{5}=\left(\begin{array}{ccc}
u_{1} & u_{2} & 0 \\
0 & u_{1} & 0 \\
0 & 0 & u_{2} \\
0 & 0 & u_{1}
\end{array}\right) \text {, } \\
& M_{6}=\left(\begin{array}{cc}
u_{1} & u_{2} \\
0 & u_{1} \\
0 & 0
\end{array}\right) \text {, } \\
& M_{n}=\left(\begin{array}{cc}
u_{1} & u_{2} \\
0 & u_{1}
\end{array}\right) \quad(n \geqslant 7) .
\end{aligned}
$$

So we get $V_{1}=V_{2}=V_{3}=\mathbb{P}_{k}^{1}, V_{4}=\left\{u_{1}=0\right\} \cup\left\{u_{2}=0\right\}$, and $V_{n}=\left\{u_{1}=0\right\}$ for $n \geqslant 5$. Hence $r=\operatorname{br}(A)=\max \left\{n \mid V_{n} \neq V_{n+1}\right\}=4$ and $J=u_{1}^{2}\left(u_{1}, u_{2}\right)$. Note that

$$
\begin{aligned}
& I_{t}\left(M_{n}\right)=\left(u_{1}, u_{2}\right)^{n}, \quad \text { if } t=1, \ldots, n(n \leqslant 3), \\
& I_{t}\left(M_{4}\right)= \begin{cases}u_{1}^{2} u_{2}^{2}, & \text { if } t=4, \\
\left(u_{1}, u_{2}\right)^{3}, & \text { if } t=3 .\end{cases}
\end{aligned}
$$

Then we can easily check that there are only two admissible sequences $(1,2,3,3)$ and $(1,2,3,4)$. 
By Theorem 1.6 we get

$$
\begin{aligned}
\operatorname{core}(A) & =\left[\left(Q_{u}, u_{1} u_{2}\right): u_{1}^{2}\left(u_{1}, u_{2}\right)^{10}\right] \cap\left[Q_{u}: u_{1}^{4} u_{2}^{2}\left(u_{1}, u_{2}\right)^{7}\right] \cap A \\
& =\left(x_{1}^{4}, x_{1}^{3} x_{2}, x_{1} x_{2}^{3}\right) .
\end{aligned}
$$

On the other hand, we have

$$
\left(Q_{u}: J^{\infty}\right) \cap A=\left(x_{1}^{4}, x_{1}^{3} x_{2}, x_{1} x_{2}^{4}\right) \neq\left(x_{1}^{4}, x_{1}^{3} x_{2}, x_{1} x_{2}^{3}\right) .
$$

One may also ask whether $\operatorname{core}(A)=Q_{u} A(u) \cap A$ holds for an arbitrary graded algebra $A$. The reason for raising this question is the fact that $Q_{u} A(u)$ is the generic minimal reduction of $A(u)$. As noted before, it has a positive answer if $A$ is a Cohen-Macaulay ring. But, as in the local case [CPU01, Example 4.11], this question has a negative answer in general.

Example 1.11. Let $A=k\left[x_{1}, x_{2}\right] /\left(x_{1} x_{2}, x_{2}^{3}\right)$. Then $\operatorname{dim} A=1$ and we can put $Q_{u}=\left(u_{1} x_{1}+u_{2} x_{2}\right)$. It is easy to check that $Q_{u} A(u) \cap A=\left(x_{1}, x_{2}\right)^{2}$. Since $x_{2}^{2} \notin\left(x_{1}\right)$, we have $x_{2}^{2} \notin \operatorname{core}(A)$. Hence $\operatorname{core}(A) \neq Q_{u} A(u) \cap A$.

Following Corso, Polini and Ulrich [CPU01, Question (iii)] one may weaken the above question to whether $\operatorname{core}(A) \subseteq Q_{u} A(u) \cap A$ for an arbitrary graded algebra $A$. Using Theorem 1.6 we can give a positive answer when $k$ is an algebraically closed field.

Corollary 1.12. Assume that $k$ is an algebraically closed field. Then

$$
\operatorname{core}(A) \subseteq Q_{u} A(u) \cap A .
$$

Proof. Let $a_{n}=\operatorname{rank} M_{n}, n=1, \ldots, r$, and $H=\left\{a_{1}, \ldots, a_{r}\right\}$. As shown in the proof of Corollary 1.9, $H$ is an admissible sequence. Note that $I_{a_{n}+1}\left(M_{n}\right)=0$ for all $n=1, \ldots, r$. Then we can deduce from Theorem 1.6 that

$$
\operatorname{core}(A) A(u) \subseteq\left(Q_{u}: J \prod_{n=1}^{r} I_{a_{n}}\left(M_{n}\right)\right) A(u)=Q_{u} A(u) .
$$

Since core $(A) \subseteq \operatorname{core}(A) A(u) \cap A$, this implies the statement.

\section{Core of ideals in local rings}

Let $(R, \mathfrak{m})$ be a local ring with infinite residue field $k$ and $I$ an ideal of $R$. Recall that an ideal $J$ is a reduction of $I$ if there exists an integer $n$ such that $J I^{n}=I^{n+1}$. The least integer $n$ with this property is called the reduction number of $I$ with respect to $J$, and we will denote it by $r_{J}(I)$. The big reduction number of $I$, denoted by $\operatorname{br}(I)$, is the supremum of all reduction numbers $r_{J}(I)$, where $J$ is a minimal reduction of $I$ with respect to inclusion.

These notions are closely related to their counterparts in the graded case. Let $F(I)=\bigoplus_{n \geqslant 0} I^{n} / \mathfrak{m} I^{n}$, the fiber ring of $I$. Then $F(I)$ is a standard graded algebra over $k$. It is known that every minimal reduction of $I$ is generated by $d$ elements [NR54], where $d=\operatorname{dim} F(I)$ (the analytic spread of $I$ ). In fact, if $c_{1}, \ldots, c_{d}$ are elements of $I$ and $c_{1}^{*}, \ldots, c_{d}^{*}$ are the residue classes of $c_{1}, \ldots, c_{d}$ in $I / \mathfrak{m} I$, then $J=\left(c_{1}, \ldots, c_{d}\right)$ is a minimal reduction of $I$ if and only if $Q=\left(c_{1}^{*}, \ldots, c_{d}^{*}\right)$ is a minimal reduction of $F(I)$. Moreover, we have $r_{J}(I)=r_{Q}(F(I))$ and, in particular, $\operatorname{br}(I)=\operatorname{br}(F(I))$ [Tru03, Lemma 4.1].

Assume that $I=\left(a_{1}, \ldots, a_{m}\right)$. Write

$$
c_{i}=\sum_{j=1}^{m} \alpha_{i j} a_{j} \quad(i=1, \ldots, d)
$$




\section{Huneke And N. V. TRung}

and put $\alpha=\left(\alpha_{i j}\right) \in R^{m d}$. We may view $J$ as a specialization at $\alpha$ of the ideal $\mathcal{Q} \subset R[u]$ generated by the generic elements:

$$
b_{i}=\sum_{j=1}^{m} u_{i j} a_{i} \quad(i=1, \ldots, d),
$$

where $u=\left\{u_{i j} \mid i=1, \ldots, d, j=1, \ldots, m\right\}$ is a family of indeterminates. The parameter space of the specializations of $\mathcal{Q}$ which are minimal reductions of $I$ can be described explicitly as follows.

Put $r=b r(I)$. Fix two minimal bases of $I^{r}$ and $I^{r+1}$ which consist of monomials in the elements $a_{1}, \ldots, a_{m}$. Write the elements of the form $b_{i} g$, where $b_{i}$ is a generic element of $I(i=1, \ldots, d)$ and $g$ is a monomial of the fixed basis of $I^{r}$, as a linear combination of the elements of the fixed basis of $I^{r+1}$ with coefficients in the polynomial ring $R[u]$. Let $M$ denote the matrix of these coefficients. Let $\mathcal{J}$ denote the ideal of $R[u]$ generated by the $(h \times h)$-minors of $M$, where $h$ is the minimal number of generators of $I^{r+1}$. It was shown in [Tru03, Proposition 4.3] that $J$ is a minimal reduction of $I$ if and only if $\mathcal{J}_{\alpha}=R$, where $\mathcal{J}_{\alpha}$ denotes the ideal of $R$ obtained from $\mathcal{J}$ by the substitution $u$ to $\alpha$.

Unlike the graded case, the above description of the parameter space of minimal reductions does not lead to a closed formula for the core. We only get the following inclusions.

Proposition 2.1. Let $\mathcal{Q}: \mathcal{J}^{\infty}$ denote the set of elements $f \in R[u]$ with $f \mathcal{J}^{n} \subseteq \mathcal{Q}$ for some positive integer $n$. Then

$$
I^{r+1} \subseteq\left[\mathcal{Q}:\left(\mathcal{Q}: I^{r+1}\right)\right] \cap R \subseteq\left[\mathcal{Q}:\left(I^{r} \mathcal{Q}: I^{r+1}\right)\right] \cap R \subseteq\left(\mathcal{Q}: \mathcal{J}^{\infty}\right) \cap R \subseteq \operatorname{core}(I) .
$$

Proof. By the definition of $\mathcal{J}$ we have $I^{r+1} \mathcal{J} \subseteq I^{r} \mathcal{Q}$. Therefore $\mathcal{J} \subseteq I^{r} \mathcal{Q}: I^{r+1} \subseteq \mathcal{Q}: I^{r+1}$. From this it follows that

$$
I^{r+1} \subseteq \mathcal{Q}:\left(\mathcal{Q}: I^{r+1}\right) \subseteq \mathcal{Q}:\left(I^{r} \mathcal{Q}: I^{r+1}\right) \subseteq \mathcal{Q}: \mathcal{J} \subseteq \mathcal{Q}: \mathcal{J}^{\infty} .
$$

It remains to show that $\left(\mathcal{Q}: \mathcal{J}^{\infty}\right) \cap R \subseteq \operatorname{core}(I)$. But this can be shown by using the above description of the parameter space of minimal reductions of $I$ (see [Tru03, Theorem 4.4]).

Corso, Polini and Ulrich [CPU01, Proposition 5.4] have shown that, if $R$ is a Cohen-Macaulay local ring and $I$ satisfies certain residual conditions (which include the case $I$ is an $\mathfrak{m}$-primary ideal), then core $(I)=\left[\mathcal{Q}:\left(\mathcal{Q}: f^{r+1}\right)\right] \cap R$ for any nonzero-divisor $f \in I$. Since $\mathcal{Q}:\left(\mathcal{Q}: f^{r+1}\right) \subseteq \mathcal{Q}:(\mathcal{Q}:$ $\left.I^{r+1}\right)$, this implies

$$
\operatorname{core}(I)=\left[\mathcal{Q}:\left(\mathcal{Q}: I^{r+1}\right)\right] \cap R=\left[\mathcal{Q}:\left(I^{r} \mathcal{Q}: I^{r+1}\right)\right] \cap R=\left(\mathcal{Q}: \mathcal{J}^{\infty}\right) \cap R .
$$

All the above facts have led to the question whether $\operatorname{core}(I)=\left(\mathcal{Q}: \mathcal{J}^{\infty}\right) \cap R$ holds in general $[$ Tru03, $\S 4]$. Using Example 1.10 we can now show that this is not the case.

Example 2.2. Let $R=k\left[x_{1}, x_{2}\right]_{\left(x_{1}, x_{2}\right)}$ where $k\left[x_{1}, x_{2}\right]:=k\left[X_{1}, X_{2}\right] /\left(X_{1}^{2} X_{2}^{2}, X_{2}^{5}\right)$. We will first show that

$$
\operatorname{core}(\mathfrak{m})=\left(x_{1}^{4}, x_{1}^{3} x_{2}, x_{1} x_{2}^{3}\right) R .
$$

Observe that $k\left[x_{1}, x_{2}\right]$ is the graded algebra $A$ considered in Example 1.10 and that $\operatorname{core}(A)=$ $\left(x_{1}^{4}, x_{1}^{3} x_{2}, x_{1} x_{2}^{3}\right)$. Since every minimal reduction of $A$ generates a minimal reduction of $\mathfrak{m}$, we have core $(\mathfrak{m}) \subseteq \operatorname{core}(A) R=\left(x_{1}^{4}, x_{1}^{3} x_{2}, x_{1} x_{2}^{3}\right) R$. For the converse inclusion, let $(c)$ be an arbitrary minimal reduction of $\mathfrak{m}$. Write $c=x+e$ with $x \in A_{1}$ and $e \in \mathfrak{m}^{2}$. Then $x$ generates a minimal reduction of core $(A)$. Hence $\left(x_{1}^{4}, x_{1}^{3} x_{2}, x_{1} x_{2}^{3}\right) \subseteq x A_{3}=(c-e) A_{3}$. Since $r_{(c)}(\mathfrak{m}) \leqslant b r(\mathfrak{m})=b r(A)=4$, we have $e A_{3} \subset \mathfrak{m}^{5} \subseteq(c)$. Therefore $(c-e) A_{3} \subset(c)$, which implies $\left(x_{1}^{4}, x_{1}^{3} x_{2}, x_{1} x_{2}^{3}\right) R \subset(c)$. So we get $\left(x_{1}^{4}, x_{1}^{3} x_{2}, x_{1} x_{2}^{3}\right) R \subseteq$ core $(\mathfrak{m})$. On the other hand, if we put $\mathcal{Q}=\left(u_{1} x_{1}+u_{2} x_{2}\right) R$, where $u_{1}, u_{2}$ are 


\section{ON THE CORE OF IDEALS}

two indeterminates, then the matrix $M$ is the matrix $M_{5}$ of Example 1.10:

$$
M=\left(\begin{array}{ccc}
u_{1} & u_{2} & 0 \\
0 & u_{1} & 0 \\
0 & 0 & u_{2} \\
0 & 0 & u_{1}
\end{array}\right)
$$

Since $\mathfrak{m}^{5}$ is generated by three elements, we get $\mathcal{J}=I_{3}(M)=u_{1}^{2}\left(u_{1}, u_{2}\right)$. Now it can be easily checked that

$$
\left(\mathcal{Q}: \mathcal{J}^{\infty}\right) \cap R=\left(x_{1}^{4}, x_{1}^{3} x_{2}, x_{1} x_{2}^{4}\right) R \neq\left(x_{1}^{4}, x_{1}^{3} x_{2}, x_{1} x_{2}^{3}\right) R=\operatorname{core}(\mathfrak{m}) .
$$

It is a natural problem to extend the results on the graded core to the core of maximal ideals in local rings which arise from graded algebras. So we raise the following basic question.

Problem. Let $(R, \mathfrak{m})$ be the localization of a standard graded algebra $A$ over an infinite field at its maximal graded ideal. Is it true that $\operatorname{core}(\mathfrak{m})=\operatorname{core}(A) R$ ?

Note that the above formula holds for the local ring of Example 2.2. Now we shall see that this formula also holds if $\operatorname{br}(A) \leqslant 2$.

Proposition 2.3. Let $(R, \mathfrak{m})$ be the localization of a standard graded algebra $A$ over an infinite field at its maximal graded ideal. Assume that $\operatorname{br}(A) \leqslant 2$. Then

$$
\operatorname{core}(\mathfrak{m})=\operatorname{core}(A) R \text {. }
$$

Proof. Since every minimal reduction of $A$ generates a minimal reduction of $\mathfrak{m}$, we always have $\operatorname{core}(\mathfrak{m}) \subseteq \operatorname{core}(A) R$. For the converse inclusion, let $J=\left(c_{1}, \ldots, c_{d}\right)$ be an arbitrary minimal reduction of $\mathfrak{m}$, where $d:=\operatorname{dim} A$. Write $c_{i}=x_{i}+e_{i}$ with $x_{i} \in A_{1}$ and $e_{i} \in \mathfrak{m}^{2}$. Let $Q$ be the ideal generated by the elements $x_{1}, \ldots, x_{d}$. Then $Q$ is a minimal reduction of $A$. Hence

$$
\operatorname{core}(A)_{2} \subseteq Q_{2} \subseteq J+\left(e_{1}, \ldots, e_{d}\right) \mathfrak{m} \subseteq J+\mathfrak{m}^{3} .
$$

Since $\operatorname{br}(\mathfrak{m})=\operatorname{br}(A)=2$, we have $\mathfrak{m}^{3} \subseteq J$. Therefore $\operatorname{core}(A)_{2} \subset J$. This implies core $(A) \subset J$, because core $(A)$ is generated by elements of degree $\geqslant 2$ unless $A$ is a polynomial ring, and $\operatorname{core}(A)_{3} \subset$ $\mathfrak{m}^{3} \subseteq J$. So we can conclude that $\operatorname{core}(A) R \subseteq \operatorname{core}(\mathfrak{m})$

Corso, Polini and Ulrich have asked whether core $\left(I R^{\prime}\right) \supseteq \operatorname{core}(I) R^{\prime}$ does hold for every flat local homomorphism $R \rightarrow R^{\prime}$ of Cohen-Macaulay local rings. We shall use the results in the graded case to construct a counter-example to this question.

Example 2.4. Let $R=\mathbb{R}\left[x_{1}, x_{2}, x_{3}, x_{4}\right]_{\left(x_{1}, x_{2}, x_{3}, x_{4}\right)}$, where

$$
\mathbb{R}\left[x_{1}, x_{2}, x_{3}, x_{4}\right]=\mathbb{R}\left[X_{1}, X_{2}, X_{3}, X_{4}\right] /\left(X_{1}^{2} X_{2}+X_{2}^{3}-X_{1}^{3} X_{3}, X_{2}^{4}-X_{1}^{4} X_{4}\right) .
$$

Then $R$ is a two-dimensional Cohen-Macaulay local ring. Let $I=\left(x_{1}, x_{2}\right)$. Since $R$ is defined by equations which are homogeneous in $x_{1}, x_{2}$, we can easily check that

$$
F(I) \cong \mathbb{R}\left[X_{1}, X_{2}\right] /\left(X_{1}^{2} X_{2}+X_{2}^{3}, X_{2}^{4}\right),
$$

which is the graded algebra of Example 1.8. From this it follows that $\operatorname{br}(I)=\operatorname{br}(F(I))=2$. Hence $I^{3} \subseteq$ core $(I)$. Moreover, every minimal reduction of $I$ is generated by an element and this element has precisely the form $x=\alpha_{1} x_{1}+\alpha_{2} x_{2}+y$ with $\alpha_{1} \neq 0$ and $y \in \mathfrak{m} I$. Thus, every element of core $(I)$ can be written both as $a x_{1}$ and as $b\left(x_{1}+x_{2}\right)$ for some elements $a, b \in R$, and we will obtain a relation of the form $a x_{1}-b\left(x_{1}+x_{2}\right)=0$. Since $R$ is defined by homogeneous equations of degree $\geqslant 3$ in $x_{1}, x_{2}$, we can easily verify that $\operatorname{core}(I)$ is contained in $I^{3}$. So we get core $(I)=I^{3}$. 


\section{Huneke And N. V. TRung}

Let $R^{\prime}=R \otimes_{\mathbb{R}} \mathbb{C}$. Similarly as above, we can also show that

$$
F\left(I R^{\prime}\right) \cong \mathbb{C}\left[X_{1}, X_{2}\right] /\left(X_{1}^{2} X_{2}+X_{2}^{3}, X_{2}^{4}\right)
$$

and that core $\left(I R^{\prime}\right)$ is contained in $I^{3} R^{\prime}$. Assume that core $\left(I R^{\prime}\right)=I^{3} R^{\prime}$. For degree reason, we must have $I^{3} R^{\prime}=J I^{2}$ for any minimal reduction $J$ of $I R^{\prime}$. By the relationship of the minimal reductions of $I R^{\prime}$ and $F\left(I R^{\prime}\right)$, this implies core $\left(F\left(I R^{\prime}\right)\right)=\left(X_{1}, X_{2}\right)^{3} F\left(I R^{\prime}\right)$. But we have seen in Example 1.8 that

$$
\operatorname{core}\left(F\left(I R^{\prime}\right)\right) \neq\left(X_{1}, X_{2}\right)^{3} F\left(I R^{\prime}\right) .
$$

So we get a contradiction. Now we can conclude that $\operatorname{core}\left(I R^{\prime}\right)$ is strictly contained in $\operatorname{core}(I) R^{\prime}=$ $I^{3} R^{\prime}$.

\section{Core of equimultiple ideals in Cohen-Macaulay local rings}

In this section we will concentrate on the core of equimultiple ideals in Cohen-Macaulay local rings with characteristic- 0 residue field. Recall that an ideal $I$ is equimultiple if the minimal reductions of $I$ are generated by $h$ elements, where $h=\mathrm{ht}(I)$. Our aim is to prove Conjecture 0.1 for this case.

First, we will consider the one-dimensional case. We shall need the following observation on the ubiquity of minimal reductions.

Lemma 3.1. Let $(R, \mathfrak{m})$ be a one-dimensional local ring with infinite residue field. Let $I$ be an $\mathfrak{m}$-primary ideal. Let $x$ be an element of $I$ which generates a minimal reduction of $I$ and $y$ an arbitrary element in $I \backslash \mathfrak{m} I$. Then we can find infinitely many units $u \in R$ with different residue classes in $R / \mathfrak{m}$ such that $(x-u y)$ is a minimal reduction of $I$.

Proof. Every element $z \in I \backslash \mathfrak{m} I$ can be represented by a point $\alpha$ in a projective space over the field $k=R / \mathfrak{m}$. Choose a set of generators $x_{1}, \ldots, x_{m}$ of $I$ and write $z=u_{1} x_{1}+\cdots+u_{m} x_{m}$, where $u_{1}, \ldots, u_{m}$ are elements of $R$. Let $\alpha_{i}$ denote the image of $u_{i}$ in the residue field $k=R / \mathfrak{m}$. By Proposition 1.1, there exists a proper projective variety $V \subset \mathbb{P}_{k}^{m-1}$ such that $(z)$ is a minimal reduction of $I$ if and only if $\left(\alpha_{1}, \ldots, \alpha_{m}\right) \notin V$. If $x, y$ are represented by the points $\alpha, \beta \in \mathbb{P}_{k}^{m-1}$, then $x-u y$ is represented by the point $\alpha-c \beta$, where $c$ denotes the image of $u$ in $k$. The conclusion follows from the fact that the line $\alpha-c \beta$ intersects $V$ only at finitely many points.

Using the above lemma we are able to characterize the core of $\mathfrak{m}$-primary ideals in a onedimensional Cohen-Macaulay local ring $R$ as follows. For a given ideal $I$ of $R$ we will denote by $B(I)$ the blow-up of $I$, that is, $B(I)=\bigcup_{n \geqslant 1}\left(I^{n}:_{F} I^{n}\right)$, where $F$ denotes the ring of fractions of $R$. The ring $B(I)$ is always in the integral closure of $R$.

Theorem 3.2. Let $(R, \mathfrak{m})$ be a one-dimensional Cohen-Macaulay local ring whose residue field has characteristic 0 . Let $I$ be an $\mathfrak{m}$-primary ideal, and let $B$ be the blow-up of $I$. Set $K$ equal to the conductor of $B$ into $R$, and let $x$ be an arbitrary minimal reduction of $I$. Then core $(I)=x K=I K$.

Proof. By [BP95, Theorem 1], we have that $I K=z K$ for every element $z \in R$ which generates a minimal reduction of $I$. This implies that $x K=I K \subseteq \operatorname{core}(I)$. Since $K=\bigcap_{r \geqslant 1}\left(x^{r}\right): I^{r}$, it remains to prove that, for any element $a x \in \operatorname{core}(I)$ and any $r \geqslant 1$, we have $a \in\left(x^{r}\right): I^{r}$. Since the residue field has characteristic $0, I^{r}$ is generated by the $r$ th powers of the elements of $I$ (see e.g. [Hun96, Exercise 1.11]). Therefore, it is sufficient to show that $a y^{r} \in\left(x^{r}\right)$ for any element $y \in I \backslash \mathfrak{m} I$. By Lemma 3.1, there exist infinitely many units $u \in R$ with different residue classes in $R / \mathfrak{m}$ such that $(x-u y)$ is a minimal reduction of $I$. For such $u$ we can write

$$
a x=b_{u}(x-u y)
$$




\section{ON THE CORE OF IDEALS}

for some element $b_{u} \in R$. Set $c=y / x$. Then $c \in B$ [Nor59, Lemma 1]. Hence, for $r$ large enough, there is an integral relation

$$
d_{0}+d_{1} c+\cdots+d_{r-1} c^{r-1}+c^{r}=0
$$

with $d_{0}, \ldots, d_{r-1} \in R$. Since $a=b_{u}(1-u c)$, we have

$$
a\left(1+u c+\cdots+u^{i-1} c^{i-1}\right)=b_{u}\left(1-u^{i} c^{i}\right)
$$

for all $i \geqslant 1$. It follows that

$$
\begin{gathered}
\sum_{i=0}^{r-1} d_{i} u^{r-i}\left[a\left(1+u c+\cdots+u^{i-1} c^{i-1}\right)\right]+a\left(1+u c+\cdots+u^{r-1} c^{r-1}\right) \\
=\sum_{i=0}^{r-1} d_{i} u^{r-i} b_{u}\left(1-u^{i} c^{i}\right)+b_{u}\left(1-u^{r} c^{r}\right)=\sum_{i=0}^{r-1} d_{i} u^{r-i} b_{u}+b_{u} \in R
\end{gathered}
$$

So we get the relation

$$
\begin{gathered}
\left(d_{0} u^{r}+d_{1} u^{r-1}+\cdots+d_{r-1} u+1\right) a+\left(d_{1} u^{r-1}+\cdots+d_{r-1} u+1\right) u a c+\cdots \\
\cdots+\left(d_{r-1} u+1\right) u^{r-2} a c^{r-2}+u^{r-1} a c^{r-1} \in R .
\end{gathered}
$$

Varying $u$ we obtain linear equations in $a, a c, \ldots, a c^{r-2}, a c^{r-1}$ modulo $R$. The rows of the coefficient matrix of $r$ such equations have the form

$$
\begin{aligned}
& \left(d_{0} u^{r}+d_{1} u^{r-1}+\cdots+d_{r-1} u+1\right),\left(d_{1} u^{r-1}+\cdots+d_{r-1} u+1\right) u, \\
& \ldots,\left(d_{r-1} u+1\right) u^{r-2}, u^{r-1} .
\end{aligned}
$$

Decomposing the $(r-1)$ th column we see that the determinant of this matrix is equal to that of the matrix of the rows

$$
\left(d_{0} u^{r}+d_{1} u^{r-1}+\cdots+d_{r-1} u+1\right),\left(d_{1} u^{r-1}+\cdots+d_{r-1} u+1\right) u, \ldots, u^{r-2}, u^{r-1} .
$$

Decomposing the other columns we will come to the Vandermonde matrix of the rows $1, u, \ldots$, $u^{r-2}, u^{r-1}$. By Lemma 3.1, we may choose the elements $u$ in such a way that the determinant of the Vandermonde matrix is a unit in $R$, and this proves that $a c, a c^{2}, \ldots, a c^{r-1} \in R$. Since $r$ can be any number large enough, we get $a c^{r} \in R$ for all $r \geqslant 1$. This implies $a y^{r} \in\left(x^{r}\right)$, as desired.

The above theorem allows us to give a positive answer to Conjecture 0.1 in the special case in which $R$ is as in the above theorem. This is due to the following set of equivalences.

Proposition 3.3. Let $(R, \mathfrak{m})$ be a one-dimensional local ring with infinite residue field, and let $I$ be an ideal containing a nonzero-divisor. Let $B$ be the blow-up of $I$ and let $K$ be the conductor of $B$ into $R$. Let $x$ be an arbitrary minimal reduction of $I$ with reduction number $r$. Then

$$
x K=\left(\left(x^{r}\right): I^{r}\right) I=\left(\left(x^{r}\right): I^{r}\right) x=\left(\left(x^{r+1}\right): I^{r}\right),
$$

and so the following are equivalent:

(i) $\operatorname{core}(I)=x K$,

(ii) $\operatorname{core}(I)=\left(\left(x^{r}\right): I^{r}\right) I$,

(iii) $\operatorname{core}(I)=\left(\left(x^{r}\right): I^{r}\right) x$,

(iv) $\operatorname{core}(I)=\left(\left(x^{r+1}\right): I^{r}\right)$.

Proof. Observe that every minimal reduction of $I$, in particular $x$, is a nonzero-divisor. We first prove that $\left(\left(x^{r}\right): I^{r}\right) I=\left(\left(x^{r}\right): I^{r}\right) x=\left(\left(x^{r+1}\right): I^{r}\right)$. Clearly $\left(\left(x^{r}\right): I^{r}\right) x \subseteq\left(\left(x^{r}\right): I^{r}\right) I$ and $\left(\left(x^{r}\right): I^{r}\right) x \subseteq\left(\left(x^{r+1}\right): I^{r}\right)$. We prove that $\left(\left(x^{r+1}\right): I^{r}\right) \subseteq\left(\left(x^{r}\right): I^{r}\right) I \subseteq\left(\left(x^{r}\right): I^{r}\right) x$ to prove all three are equal. Let $s \in\left(\left(x^{r+1}\right): I^{r}\right)$. Then, in particular, $s x^{r}=t x^{r+1}$ for some $t \in R$, so that 


\section{Huneke And N. V. TRung}

as $x$ is a nonzero-divisor, $s=x t$. As $x t I^{r} \subseteq\left(x^{r+1}\right)$, we have that $t \in\left(\left(x^{r}\right): I^{r}\right)$, showing that $s \in\left(\left(x^{r}\right): I^{r}\right) x \subseteq\left(\left(x^{r}\right): I^{r}\right) I$. Finally, let $u \in\left(\left(x^{r}\right): I^{r}\right) I$. Then $x^{r-1} u \in\left(x^{r}\right)$ which forces $u=x v$ for some $v \in R$. But then $x v I^{r} \subseteq I^{r} \cdot I\left(\left(x^{r}\right): I^{r}\right)=x I^{r}\left(\left(x^{r}\right): I^{r}\right)$ (because $\left.I^{r+1}=x I^{r}\right)$, so that $v \in\left(\left(x^{r}\right): I^{r}\right)$, and $u \in\left(\left(x^{r}\right): I^{r}\right) x$.

It remains to prove that $x K$ is equal to any of the other three ideals. We prove that $K=$ $\left(\left(x^{r}\right): I^{r}\right)$. If $b \in K$, then $b I^{n} \subseteq\left(x^{n}\right)$ for all $n \geqslant 1$, since $I^{n} / x^{n} \subseteq B$. Conversely, if $w \in\left(\left(x^{r}\right): I^{r}\right)$, then we claim that $w \in K$. Let $b \in B$. We can write $b=a / x^{n}$ for some $a \in I^{n}$. Without loss of generality we can assume that $n \geqslant r+1$. Then $w a \in w I^{n} \subseteq w x^{n-r} I^{r} \subseteq\left(x^{n}\right)$, which implies that $w b \in R$. Thus $K=\left(\left(x^{r}\right): I^{r}\right)$, finishing the proof.

Corollary 3.4. Let $(R, \mathfrak{m})$ be a one-dimensional Cohen-Macaulay local ring whose residue field has characteristic 0 . Let $I$ be an $\mathfrak{m}$-primary ideal of $R$, let $x$ be a minimal reduction of $I$, and let $r$ be the reduction number of $I$ with respect to $x$. Then

$$
\operatorname{core}(I)=\left(\left(x^{r}\right): I^{r}\right) I=\left(x^{r}: I^{r}\right) x=\left(\left(x^{r+1}\right): I^{r}\right) .
$$

Proof. The corollary follows at once from Theorem 3.2 and Proposition 3.3.

It is easy to find examples which show that Corollary 3.4 does not hold if the $\operatorname{ring} A$ is not Cohen-Macaulay.

Example 3.5. Let $A=k\left[x_{1}, x_{2}\right]_{\left(x_{1}, x_{2}\right)}$ with $k\left[x_{1}, x_{2}\right]:=k\left[X_{1}, X_{2}\right] /\left(X_{1} X_{2}, X_{2}^{2}\right)$. Let $I$ be the maximal ideal of $A$. Then $\left(x_{1}\right)$ is a minimal reduction of $I$ with reduction number 1 . We have $\left(x_{1}^{2}\right): I=$ $\left(x_{1}, x_{2}\right)$. Since $x_{2}$ is not contained in $\left(x_{1}\right)$, we must have core $(I) \neq\left(x_{1}^{2}\right): I$.

To pass from the one-dimensional case to the general case we shall need the following result of Hyry and Smith.

Lemma 3.6 [HS02, Lemma 5.1.3]. Let $R$ be a Cohen-Macaulay local ring. Let $I$ be an equimultiple ideal of $R$ with $h=\operatorname{ht}(I) \geqslant 1$, let $J$ be a minimal reduction of $I$, and let $r$ be the reduction number of $I$. Then the ideal $J^{r+1}: I^{r}$ does not depend on the choice of $J$.

Now we are able to settle Conjecture 0.1 for equimultiple ideals in Cohen-Macaulay local rings whose residue field has characteristic 0.

Theorem 3.7. Let $R$ be a Cohen-Macaulay local ring whose residue field has characteristic 0. Let $I$ be an equimultiple ideal of $R$ with $h=\mathrm{ht}(I) \geqslant 1$, let $J$ be a minimal reduction of $I$, and let $r$ be the reduction number of $I$. Then

$$
\operatorname{core}(I)=J^{r+1}: I^{r}
$$

Proof. Since $J$ is generated by a regular sequence, we have $J^{r+1}: I^{r} \subseteq J^{r+1}: J^{r}=J$. Since $J^{r+1}: I^{r}$ does not depend on $J$ by Lemma 3.6, this implies $J^{r+1}: I^{r} \subseteq \operatorname{core}(I)$. It remains to show that $\operatorname{core}(I) \subseteq J^{r+1}: I^{r}$. This inclusion is equivalent to the formula

$$
\operatorname{core}(I) \subseteq J^{n+1}: I^{n}
$$

for all $n \geqslant 0$. In fact, we always have $J^{r+1}: I^{r} \subseteq J^{r+1}: J^{r-n} I^{n}=J^{n+1}: I^{n}$ for $n \leqslant r$ and $J^{n+1}: I^{n}=J^{n+1}: J^{n-r} I^{r}=J^{r+1}: I^{r}$ for $n>r$. We will use induction on $n$ and $h$ to prove the above formula.

If $n=0$, it is trivial because core $(I) \subseteq J=J: I^{0}$. If $h=1$, we will go back to the onedimensional case. Let $\operatorname{Min}\left(J^{r+1}\right)$ denote the set of the minimal associated primes of $J^{r+1}$. Since $J^{r+1}$ is an unmixed ideal, we have

$$
J^{r+1}=\bigcap_{P \in \operatorname{Min}\left(J^{r+1}\right)} J^{r+1} A_{P} \cap A .
$$




\section{ON THE CORE OF IDEALS}

By [CPU01, Theorem 4.5] we have

$$
\operatorname{core}(I) \subseteq \bigcap_{P \in \operatorname{Min}\left(J^{r+1}\right)} \operatorname{core}\left(I_{P}\right) \cap A .
$$

Since $\operatorname{dim} A_{P}=1$, we may apply Corollary 3.4 to $\operatorname{core}\left(I_{P}\right)$ and obtain

$$
\begin{aligned}
\operatorname{core}(I) & \subseteq \bigcap_{P \in \operatorname{Min}\left(J^{r+1}\right)}\left(J^{r+1} A_{P}: I^{r}\right) \cap A \\
& \subseteq\left(\bigcap_{P \in \operatorname{Min}\left(J^{r+1}\right)} J^{r+1} A_{P} \cap A\right): I^{r}=J^{r+1}: I^{r} .
\end{aligned}
$$

As noted above, this implies the formula core $(I) \subseteq J^{n+1}: I^{n}$ for all $n \geqslant 0$ when $h=1$.

Now let $n>0$ and $h>1$. Using induction on $n$ we may assume that $\operatorname{core}(I) \subseteq J^{n}: I^{n-1}$. Let $S$ denote the set of all superficial elements of $I$ which belong to minimal bases of $J$. Note that all elements of $S$ are necessarily nonzero-divisors. For an arbitrary $x \in S$ let $\bar{R}=R /(x), \bar{I}=I /(x)$ and $\bar{J}=J /(x)$. Then $\bar{A}$ is a Cohen-Macaulay local $\operatorname{ring}, \bar{I}$ is an equimultiple ideal with $h t(\bar{I})=h-1$, and $\bar{J}$ is a minimal reduction of $\bar{I}$. Using induction on $h$ we may assume that $\operatorname{core}(\bar{I}) \subseteq \bar{J}^{n+1}: \bar{I}^{n}$. Let $a_{1}, \ldots, a_{h-1}$ be elements of $I$ such that $\left(a_{1}, \ldots, a_{h-1}, x\right) /(x)$ is a minimal reduction of $\bar{I}$. Then $I^{n+1} \subseteq\left(a_{1}, \ldots, a_{h-1}\right) I^{n}+(x)$ for $n \gg 0$. Since $x$ is a superficial element of $I$, there exists an integer $c$ such that $\left(I^{n+1}: x\right) \cap I^{c}=I^{n}$ for $n \gg 0$. By the Artin-Rees lemma, $(x) \cap I^{n+1} \subseteq x I^{n-n_{0}}$ for some integer $n_{0}$. Hence

$$
(x) \cap I^{n+1}=x\left[\left(I^{n+1}: x\right) \cap I^{n-n_{0}}\right]=x\left[\left(I^{n+1}: x\right) \cap I^{c} \cap I^{n-n_{0}}\right]=x I^{n}
$$

for $n \gg 0$. These facts imply

$$
I^{n+1}=\left(a_{1}, \ldots, a_{h-1}\right) I^{n}+(x) \cap I^{n+1}=\left(a_{1}, \ldots, a_{h-1}, x\right) I^{n}
$$

for $n \gg 0$. So $\left(a_{1}, \ldots, a_{h-1}, x\right)$ is a minimal reduction of $I$. Now it is clear that $\operatorname{core}(\bar{I})=\bigcap K /(x)$, where $K$ runs through all minimal reductions of $I$ containing $x$. Therefore, $\bigcap K \subseteq\left(J^{n+1}, x\right):\left(I^{n}, x\right)$. Since core $(I) \subseteq \bigcap K$, we get

$$
\operatorname{core}(I) I^{n} \subseteq J^{n} \cap\left(J^{n+1}, x\right)=x J^{n-1}+J^{n+1} .
$$

Let $G=\bigoplus_{t \geqslant 0} J^{t} / J^{t+1}$ be the associated graded ring of $J$. Then $G$ is a Cohen-Macaulay ring with $\mathrm{ht}\left(G_{+}\right) \geqslant 2$. Since $S$ corresponds to the complement of a finite union of linear subspaces of $J / \mathfrak{m} J$, we can choose elements $x_{1}, \ldots, x_{n+1} \in S$ such that their initial forms $x_{1}^{*}, \ldots, x_{n+1}^{*}$ in $G$ are pairwise relatively prime. We have $\left(x_{1}^{*}\right) \cap \cdots \cap\left(x_{n+1}^{*}\right)=\left(x_{1}^{*} \ldots x_{n+1}^{*}\right)$. Since $\left(x_{1}^{*} \ldots x_{n+1}^{*}\right)_{n}=0$, we get

$$
\operatorname{core}(I) \subseteq\left(x_{1} J^{n-1}+J^{n+1}\right) \cap \cdots \cap\left(x_{n+1} J^{n-1}+J^{n+1}\right)=J^{n+1} .
$$

This proves the formula core $(I) \subseteq J^{n+1}: I^{n}$ for all $n>0$ and $h>1$.

\section{ACKNOWLEDGEMENTS}

Part of the work of this paper was done when the second author visited Lawrence, Kansas, in the fall of 2001. He would like to thank the Department of Mathematics of the University of Kansas for support and hospitality. Both authors thank the referee for a careful reading of this paper.

\section{REFERENCES}

BP95 V. Barucci and K. Pettersson, On the biggest maximally generated ideal as the conductor in the blowing up ring, Manuscripta Math. 88 (1995), 457-466.

CPU01 A. Corso, C. Polini and B. Ulrich, The structure of the core of ideals, Math. Ann. 321 (2001), 89-105. 


\section{ON THE CORE OF IDEALS}

CPU02 A. Corso, C. Polini and B. Ulrich, Core and residual intersections of ideals, Trans. Amer. Math. Soc. 354 (2002), 2579-2594.

HS95 C. Huneke and I. Swanson, Cores of ideals in 2-dimensional regular local rings, Michigan Math. J. 42 (1995), 193-208.

HS02 E. Hyry and K. E. Smith, On a non-vanishing conjecture of Kawamata and the core of an ideal, Amer. J. Math. 125 (2003), 1349-1410.

Hun96 C. Huneke, Tight closures and its applications, CBMS Regional Conference Series in Mathematics, vol. 88 (American Mathematical Society, Providence, RI, 1996).

Nor59 D. G. Northcott, On the notion of a first neighbourhood ring, Proc. Cambridge Philos. Soc. 53 (1959), 43-56.

NR54 D. G. Northcott and D. Rees, Reductions of ideals in local rings, Proc. Cambridge Philos. Soc. 50 (1954), 145-158.

PU04 C. Polini and B. Ulrich, A formula for the core of an ideal, Math. Ann., to appear.

RS88 D. Rees and J. Sally, General elements and joint reductions, Michigan Math. J. 35 (1988), 241-254.

Tru87 N. V. Trung, Reduction exponent and degree bound for the defining equations of graded rings, Proc. Amer. Math. Soc. 101 (1987), 229-236.

Tru03 N. V. Trung, Constructive characterizations of the reduction numbers, Compositio Math. 137 (2003), 99-113.

Vas96 W. Vasconcelos, The reduction number of an algebra, Compositio Math. 106 (1996), 189-197.

Vas99 W. Vasconcelos, Reduction numbers of ideals, J. Algebra 216 (1999), 652-664.

Craig Huneke huneke@math.ukans.edu

Department of Mathematics, University of Kansas, Lawrence, KS 66045-7523, USA

Ngô Viêt Trung nvtrung@math.ac.vn

Institute of Mathematics, Box 631, Bò Hô, 10000 Hanoi, Vietnam 